Original Article

Journal of Epilepsy Research pISSN 2233-6249 / eISSN 2233-6257

\title{
Factors Affecting Cognition and Depression in Adult Patients with Epilepsy
}

Jaishree Narayanan, MD¹, Kelly Claire Simon, $\mathrm{SCD}^{1}$, Janet Choi, MD¹, Sofia Dobrin, MD', Susan Rubin, MD', Jesse Taber, MD', Charles Wang, MD , Anna Pham ', Richard Chesis, BBA², Bryce Hadsell, MS², Alexander Epshteyn, MPM², Gary Wilk, PhD², Samuel Tideman, MS², Steven Meyers, $\mathrm{MD}^{1}$, Roberta Frigerio, $\mathrm{MD}^{1}$, Demetrius Maraganore ${ }^{3}$

${ }^{1}$ NorthShore Neurological Institute, ${ }^{2}$ Health Information Technology, NorthShore University HealthSystem, Evanston, IL; ${ }^{3}$ Department of Neurology, University of Florida, Gainesville, FL, USA

Background and Purpose: Epilepsy patients are more likely to experience depressive symptoms and cognitive impairment compared to individuals in the general population. As the reasons for this are not definitively known, we sought to determine what factors correlate most strongly with cognition and a screening test for depression in epilepsy patients.

Methods: Our study population included 379 adult patients diagnosed with epilepsy or seizure in our neurology clinic. We collected detailed demographic and clinical data during patient visits using structured clinical documentation support tools that we have built within our commercial electronic medical records system (Epic), including a depression score (Neurological Disorders Depression Inventory for Epilepsy, NDDIE) and cognition score test measures (specifically in this study, Mini-Mental State Examination [MMSE]). Medication, age, gender, body mass index, duration of epilepsy, seizure frequency, current number of anti-epileptic medications, years of education were assessed in relation to baseline score as well as change in score from initial visit to first annual follow-up.

Results: Of the analyzed factors, two statistically significant associations were found after correction for multiple testing. Male gender and lower anti-seizure medication count were associated with better mood, as assessed by NDDIE score, at initial visit. Specifically, male gender was associated with a 1.3 decrease in NDDIE and for each additional anti-seizure medication, there was an associated 1.2 increase in NDDIE. Conclusions: However, these factors were not associated with change in NDDIE score from initial to first annual follow-up visit. These findings, although interesting, are preliminary. Additionally, these findings were based on a homogenous (mainly Caucasian) clinic-based population and detailed information on previous medication use was lacking. Further work is needed to replicate these findings and to understand any mechanisms that may explain these associations. (2019;9:103-110)

Key words: Epilepsy, Cognitive dysfunction, Depression, Documentation
Received November 22,2019

Revised January 24, 2020

Corresponding author:

Jaishree Narayanan, MD

NorthShore Neurological Institute,

NorthShore University HealthSystem, 2650

Ridge Ave., Evanston, IL 60201, USA

Tel. +1-847-570-1678

Fax. $+1-847-733-5565$

E-mail; jnarayanan@northshore.org

\section{Introduction}

Epilepsy is a relatively common neurological disorder. Approximately, 70 million people have epilepsy globally. Epilepsy incidence is generally bimodal with peaks in younger and older age groups. ${ }^{1}$ The clinical course and severity of epilepsy are variable depending on age, co-morbidities, and other factors. ${ }^{1}$ For patients with more severe or treatment-refractory epilepsy, there is a significant impact on quality of life. Not surprisingly, depression is more common in patients with epilepsy than in the general population of same-aged healthy individuals, ${ }^{2}$ and a bidirectional shared neurobiology has been proposed. ${ }^{3}$ However, there is considerable variability among epilepsy patients' frequency and severity of depression. Interestingly, it is not clear if it is entirely dependent on quality of life, suggesting other factors may be important in predicting the risk of depression in these patients. Similarly, cognitive impairment is more common in epilepsy patients compared to same-age healthy controls. ${ }^{2}$ Because both cognitive impairment and depression can have significant effects on qual- 
ity of life, it is important to understand what factors may predict mood and cognitive impairment, particularly if there are modifiable factors or factors that would benefit from early intervention. The relationship between mood and cognitive impairment in epilepsy is likely multifactorial. Possible explanations include shared neurobiology, comorbidities, medication effects, and socio-economic factors, among others. ${ }^{4-13}$ Understanding these relationships will improve the care given to patients with epilepsy, who already have a condition which imposes a significant impact on their quality of life.

The aim of our study is to determine factors that have significant association with depression and cognitive impairment in patients with epilepsy. The study design involves a point of care structured data collection from patients with epilepsy in the epilepsy clinic during their office visit and analysis of the data collected to address the study aim.

\section{Methods}

\section{Study population}

Our study population included all adult patients (ages 18 and above) who were seen at the epilepsy clinic for their initial visit at Northshore University HealthSystem between January 21, 2014 and April 12, 2019, and had a final diagnosis of seizure or epilepsy. We identified 666 patients who met these criteria during the study period. We restricted our analyses to patients with the relevant outcome measure documented (total number in regression for Neurological Disorders Depression Inventory for Epilepsy [NDDIE] was 377 and for Mini-Mental State Examination [MMSE] was 369). Of these patients, a sub-set have returned for first annual follow-up visits and are included in the longitudinal analyses (190 for analysis of NDDIE and 189 for analysis of MMSE).

\section{Design methods}

Data was collected at the point of care during the outpatient clinic visit, using structured clinical documentation support tools that we have built within our commercial electronic medical records (EMR) system (Epic). ${ }^{14,15}$ The tools navigate care according to best practices, capture several hundred fields of epilepsy data per office visit and write notes at initial and annual follow-up visits. A validated screening tool for depressive symptoms was administered using the NDDIE. ${ }^{16}$ The 6 -item NDDIE was used as validated by Gilliam et al. ${ }^{16}$ A score of 15 had a specificity of $90 \%$, a sensitivity of $81 \%$, and a positive predictive value of 0.62 for a diagnosis of major depression. ${ }^{16}$ Therefore, a score of 15 was used as the cut-off for major depression.

Initially, for cognitive assessment, we used the Montreal Cognitive Assessment (MoCA). ${ }^{17}$ However, due to licensing permissions, the burden to the nursing staff and physician request, we switched to the Short Test of Mental Status (STMS). STMS was used as defined by Kokmen et al. ${ }^{17}$ A score of 29 (out of a maximum of 38 ) or less is considered screening positive for dementia. The reported sensitivity was 92\% and specificity was $91 \% .{ }^{17}$ Because both scores (MoCA and STMS) can be converted to the MMSE using published nomograms ${ }^{18}$ for MOCA and unpublished nomograms for STMS. For comparability, we present all scores as their MMSE converted score. For MMSE, scores of 23 or less are commonly considered abnormal. ${ }^{19}$ We chose other variables of interest based on a priori knowledge from published literature, ${ }^{4-13}$ including age (years), gender, body mass index $\left(\mathrm{BMI} ; \mathrm{kg} / \mathrm{m}^{2}\right)$, use of commonly prescribed epilepsy medications (levetiracetam, lamotrigine, valproic acid and carbamazepine), years of education, duration of epilepsy, seizure frequency (defined as number of seizures per day), and number of current anti-epileptic drugs (AEDs). In addition, we screened for commonly prescribed anti-depressant medications (fluoxetine, paroxetine, sertraline, citalopram, escitalopram, and venlafaxine). Medication use was determined from the physician's review of the patient's medications at each office visit. We dichotomized anti-depressant medication use based on whether a patient reported current use of one of the listed anti-depressant medications.

\section{Statistical methods}

Multivariable linear regression models were used to assess the linear association between NDDIE or MMSE, age, gender, medications, duration of epilepsy, seizure frequency, number of current anti-seizure medications, years of education and BMI. The open source statistical software R (version 3.3.2; Microsoft, Seattle, WA, USA) was used for all analyses. We conducted two sets of analyses, and the first considered the association between the covariates and MMSE or NDDIE at initial baseline visit. In longitudinal analyses, we further adjusted for baseline NDDIE or MMSE score and assessed the association between the covariates at baseline and change in NDDIE or MMSE from initial to first annual visit. To assess for potential confounding by anti-depressant medication use, we ran baseline and longitudinal analyses with and without anti-depressant medications and compared the point estimates of variables of interest between the two models. Because this was an exploratory study, we used a 
Bonferroni correction to determine statistical significance. $p$-values were considered significant if $p<0.0045$ ( 0.05 divided by 11 tests). We restricted our analyses to patients with the relevant outcome measure documented (total number in regression for NDDIE was 377 and for MMSE was 369). Of these patients, a sub-set have returned for first annual visits and are included in the longitudinal analyses (190 for analysis of NDDIE and 189 for analysis of MMSE).

\section{Results}

Baseline characteristics of our study population are shown in Table 1. Results of multivariable regression analyses assessing the relationship between age, BMl, gender, medication use, epilepsy duration, epilepsy severity, and years of education with NDDIE or MSSE are shown in Table 2 for initial visit and Table 3 for change from initial to first annual follow-up visit. In addition, multivariable regression analyses including anti-depressant medication use for the initial visit and the change from initial to annual follow-up visits are included in Tables 4 and 5, respectively. The time between the first annual follow-up visit and initial visit ranged from 9-15 months.

\section{NDDIE}

In multivariable models, for the association with baseline NDDIE, we found that male gender ( $p_{\text {uncorrected }}=0.004$ ) and lower number of anti-seizure medications ( $p_{\text {uncorrected }}=0.001$ ) were associated with better mood after adjustment for multiple comparisons. Specifically, men had on average, NDDIE scores that were 1.3 points lower than women. For AED count, use of each additional AED medication (increasing count) was associated with a 1.2 increase in NDDIE. None of the medications (lamotrigine, valproic acid, levetiracetam, and carbamazepine) analyzed had a significant association with mood. For change in NDDIE, there was an association with valproic acid use. Use of valproic acid was associated with a higher NDDIE score (1.9 points). However, this was no longer significant after correction for multiple testing (Puncorrected $>0.0045$ ). None of the other factors studied showed a statistically significant association with change in NDDIE scores including levetiracetam, lamotrigine and carbamazepine.

\section{MMSE}

As expected, there was a significant association between ages ( puncorrected $=0.002$ ), with each increasing year of age being associated with a -0.02 decrease in MMSE score. Additionally, number of years of education and MMSE scores were strongly associated (puncorrected $<0.001$ ). Each additional year of education was associated with a 0.28 increase in MMSE. The other significant association was between MMSE score and seizure frequency ( $p_{\text {uncorrected }}=0.03$ ). Patients with more frequent seizures had a significantly lower cognitive status. However, this was no longer significant after adjustment for multiple testing. No other significant associations were observed. For the change in MMSE scores from initial visit to first annual fol-

Table 1. Descriptive characteristics for 385 epilepsy patients included in analysis

\begin{tabular}{|c|c|}
\hline & Value $(n=385)$ \\
\hline Age at encounter & $45(18-94)$ \\
\hline Age at onset & $27(0-91)$ \\
\hline Age at diagnosis & $26(0-93)$ \\
\hline Disease duration & $7.8(0-70)$ \\
\hline Female & $203(52)$ \\
\hline \multicolumn{2}{|l|}{ Race } \\
\hline Caucasian & $306(80)$ \\
\hline African-American & $34(9)$ \\
\hline Hispanic/Latino & $26(7)$ \\
\hline Asian & $18(5)$ \\
\hline American Indian or Alaskan Native & $1(<1)$ \\
\hline $\mathrm{BMl}$ & $26(17-659)$ \\
\hline Years of education & $16(6-30)$ \\
\hline \multicolumn{2}{|l|}{ Smoking history } \\
\hline Never & $235(61)$ \\
\hline Former & $111(29)$ \\
\hline Current & $38(10)$ \\
\hline Passive & $1(<1)$ \\
\hline $\begin{array}{l}\text { NDDIE: signs or symptoms of major } \\
\text { depression, cutoff score } 15\end{array}$ & $79(21)$ \\
\hline NDDIE score & $11(6-24)$ \\
\hline $\begin{array}{l}\text { MMSE: possible cognitive impairment, score } \\
\text { of }>23 \text { abnormal }\end{array}$ & $21(6)$ \\
\hline MMSE score & $28(10-30)$ \\
\hline \multicolumn{2}{|l|}{ Anti-epileptic drugs } \\
\hline Levetiracetam & $182(47)$ \\
\hline Lamotrigine & $52(14)$ \\
\hline Valproic acid & $49(13)$ \\
\hline Carbamazepine & $32(8)$ \\
\hline Monotherapy & $268(70)$ \\
\hline Polytherapy & $90(23)$ \\
\hline Seizure frequency per day & $0.001(0.0003-0.57)$ \\
\hline Anti-depressant drugs & $16(4)$ \\
\hline
\end{tabular}

Values are presented as median (range) or number (\%). BMI, body mass index; NDDIE, Neurological Disorders Depression Inventory for Epilepsy; MMSE, Mini-Mental State Examination. 
low-up visit, there was suggestion of an association with BMI ( unncorrected $=0.008$ ). Each one unit increase in BMI was associated with a 0.06 decrease in MMSE. However, this association was no longer significant after correction for multiple testing. No other significant associations were observed.

\section{Discussion}

The structured data collection for epilepsy toolkit that we have incorporated into our EMR system (Epic) offers a powerful way of collecting several hundred fields of epilepsy related data per office visit, which allows for conducting research conduct research at the point-of-care. ${ }^{15}$ We sought to investigate the correlation between se-

Table 2. p-values from linear regression of baseline NDDIE or MMSE and age, gender, BMI, medication use, seizure frequency, number of anti-seizure medications, duration of epilepsy, and years of education at baseline

\begin{tabular}{|c|c|c|c|c|c|c|}
\hline & \multicolumn{3}{|c|}{ NDDIE $(n=377)$} & \multicolumn{3}{|c|}{ MSSE $(n=369)$} \\
\hline & Coefficients & SE & $p$-value & Coefficients & SE & $p$-value \\
\hline Age & -0.013 & 0.013 & 0.33 & -0.023 & 0.007 & $0.002^{*}$ \\
\hline BMl & 0.072 & 0.036 & 0.047 & -0.011 & 0.021 & 0.59 \\
\hline Gender & -1.34 & 0.45 & $0.003^{*}$ & -0.065 & 0.26 & 0.81 \\
\hline Years of education & -0.08 & 0.078 & 0.31 & 0.28 & 0.046 & $<0.00001^{*}$ \\
\hline Disease duration & -0.019 & 0.016 & 0.25 & -0.004 & 0.009 & 0.68 \\
\hline \# AEDs & 1.24 & 0.37 & $0.001 *$ & -0.22 & 0.22 & 0.33 \\
\hline Seizure frequency & 3.18 & 3.25 & 0.33 & -4.04 & 1.86 & 0.03 \\
\hline Levetiracetam & -0.92 & 0.55 & 0.1 & 0.0004 & 0.32 & 0.999 \\
\hline Lamotrigine & -0.31 & 0.72 & 0.67 & 0.14 & 0.42 & 0.73 \\
\hline Valproic acid & -0.57 & 0.77 & 0.46 & -0.84 & 0.46 & 0.067 \\
\hline Carbamazepine & -1.47 & 0.85 & 0.08 & 0.67 & 0.50 & 0.18 \\
\hline
\end{tabular}

p-values shown in table are uncorrected values. Significant after correction for multiple testing AED.

NDDIE, Neurological Disorders Depression Inventory for Epilepsy; MMSE, Mini-Mental State Examination; BMI, body mass index; SE, standard error; AEDs, anti-epileptic drugs.

${ }^{*} p$-values that are significant after multiple hypothesis correction.

Table 3. $p$-values from linear regression for change in NDDIE or MMSE from initial to annual follow-up visit

\begin{tabular}{|c|c|c|c|c|c|c|}
\hline & \multicolumn{3}{|c|}{ NDDIE $(n=190)$} & \multicolumn{3}{|c|}{ MMSE $(n=189)$} \\
\hline & Coefficients & SE & $p$-value & Coefficients & SE & $p$-value \\
\hline Initial score & -0.38 & 0.055 & $<0.00001^{*}$ & -0.27 & 0.057 & $<0.0001$ * \\
\hline Age & 0.01 & 0.014 & 0.50 & -0.005 & 0.009 & 0.60 \\
\hline BMl & 0.02 & 0.04 & 0.63 & -0.065 & 0.024 & 0.008 \\
\hline Gender & -0.47 & 0.50 & 0.34 & 0.22 & 0.30 & 0.47 \\
\hline Years of education & -0.14 & 0.08 & 0.08 & 0.072 & 0.05 & 0.17 \\
\hline Disease duration & -0.03 & 0.017 & 0.08 & 0.014 & 0.01 & 0.15 \\
\hline \# AEDs & -0.57 & 0.41 & 0.16 & -0.39 & 0.26 & 0.14 \\
\hline Seizure frequency & -4.70 & 3.21 & 0.14 & 0.52 & 1.90 & 0.79 \\
\hline Levetiracetam & -0.35 & 0.59 & 0.55 & -0.084 & 0.36 & 0.81 \\
\hline Lamotrigine & 0.21 & 0.78 & 0.79 & -0.33 & 0.46 & 0.48 \\
\hline Valproic acid & 1.92 & 0.84 & 0.02 & 0.95 & 0.55 & 0.08 \\
\hline Carbamazepine & -0.31 & 0.85 & 0.71 & -0.30 & 0.52 & 0.57 \\
\hline
\end{tabular}

$p$-values shown in table are uncorrected $p$-values. Significant after correction for multiple testing AED.

NDDIE, Neurological Disorders Depression Inventory for Epilepsy; MMSE, Mini-Mental State Examination; BMI, body mass index; SE, standard error; AEDs, anti-epileptic drugs.

${ }^{*} p$-values that are significant after multiple hypothesis correction. 
lect patient characteristics, and NDDIE or MMSE scores. We analyzed the association between age, gender, BMI, levetiracetam, lamotrigine, valproic acid, carbamazepine, duration of epilepsy, seizure frequency, years of education, current number of anti-seizure medi- cations and NDDIE and MMSE scores at initial visit and over time. Duration of each medication use was not included since the information was collected during the office visit from patient reports and many of the patients were not sure about how long they had

Table 4. $p$-values from linear regression of baseline NDDIE or MMSE and age, gender, BMI, medication use, seizure frequency, number of anti-seizure medications, duration of epilepsy, years of education and anti-depressant medications at baseline

\begin{tabular}{|c|c|c|c|c|c|c|}
\hline & \multicolumn{3}{|c|}{ NDDIE $(n=377)$} & \multicolumn{3}{|c|}{ MSSE $(n=369)$} \\
\hline & Coefficients & SE & $p$-value & Coefficients & SE & $p$-value \\
\hline Age & -0.011 & 0.013 & 0.38 & -0.023 & 0.007 & $0.002^{*}$ \\
\hline BMl & 0.067 & 0.036 & 0.06 & -0.011 & 0.021 & 0.60 \\
\hline Gender & -1.33 & 0.45 & $0.003^{*}$ & -0.064 & 0.26 & 0.81 \\
\hline Years of education & -0.068 & 0.077 & 0.38 & 0.28 & 0.046 & $<0.00001$ * \\
\hline Disease duration & -0.02 & 0.016 & 0.21 & -0.004 & 0.009 & 0.69 \\
\hline \# AEDs & 1.31 & 0.37 & $0.0004^{*}$ & -0.22 & 0.22 & 0.33 \\
\hline Seizure frequency & 2.24 & 3.21 & 0.49 & -4.00 & 1.87 & 0.03 \\
\hline Levetiracetam & -0.97 & 0.54 & 0.07 & 0.002 & 0.32 & 0.997 \\
\hline Lamotrigine & -0.52 & 0.71 & 0.46 & 0.15 & 0.42 & 0.72 \\
\hline Valproic acid & -0.70 & 0.76 & 0.35 & -0.84 & 0.46 & 0.069 \\
\hline Carbamazepine & -1.74 & 0.84 & 0.04 & 0.68 & 0.50 & 0.18 \\
\hline Anti-depressant & 3.87 & 1.08 & $0.0004^{*}$ & -0.14 & 0.65 & 0.84 \\
\hline
\end{tabular}

p-values shown in table are uncorrected values. Significant after correction for multiple testing AED.

NDDIE, Neurological Disorders Depression Inventory for Epilepsy; MMSE, Mini-Mental State Examination; BMI, body mass index; SE, standard error; AEDs, anti-epileptic drugs.

${ }^{*} p$-values that are significant after multiple hypothesis correction.

Table 5. p-values from linear regression for change in NDDIE or MMSE from initial to annual follow-up visit including anti-depressant medications

\begin{tabular}{|c|c|c|c|c|c|c|}
\hline & \multicolumn{3}{|c|}{ NDDIE $(n=190)$} & \multicolumn{3}{|c|}{ MMSE $(n=189)$} \\
\hline & Coefficients & SE & $p$-value & Coefficients & SE & $p$-value \\
\hline Initial score & -0.38 & 0.056 & $<0.00001 *$ & -0.27 & 0.057 & $<0.0001$ * \\
\hline Age & 0.01 & 0.014 & 0.50 & -0.005 & 0.008 & 0.60 \\
\hline $\mathrm{BMl}$ & 0.02 & 0.04 & 0.63 & -0.065 & 0.025 & 0.009 \\
\hline Gender & -0.47 & 0.50 & 0.35 & 0.22 & 0.30 & 0.47 \\
\hline Years of education & -0.14 & 0.08 & 0.08 & 0.072 & 0.05 & 0.17 \\
\hline Disease duration & -0.03 & 0.017 & 0.08 & 0.014 & 0.01 & 0.15 \\
\hline \# AEDs & -0.57 & 0.41 & 0.17 & -0.39 & 0.26 & 0.15 \\
\hline Seizure frequency & -4.71 & 3.22 & 0.15 & 0.52 & 1.91 & 0.79 \\
\hline Levetiracetam & -0.35 & 0.59 & 0.55 & -0.086 & 0.36 & 0.81 \\
\hline Lamotrigine & 0.21 & 0.78 & 0.79 & -0.33 & 0.47 & 0.47 \\
\hline Valproic acid & 1.92 & 0.85 & 0.02 & 0.95 & 0.55 & 0.08 \\
\hline Carbamazepine & -0.31 & 0.87 & 0.73 & -0.30 & 0.53 & 0.56 \\
\hline Anti-depressant & -0.06 & 1.17 & 0.96 & 0.06 & 0.73 & 0.94 \\
\hline
\end{tabular}

$p$-values shown in table are uncorrected $p$-values. Significant after correction for multiple testing AED.

NDDIE, Neurological Disorders Depression Inventory for Epilepsy; MMSE, Mini-Mental State Examination; BMI, body mass index; SE, standard error; AEDs, anti-epileptic drugs.

* $p$-values that are significant after multiple hypothesis correction. 
used a specific medication. After correction for multiple comparisons, two statistically significant findings were observed between baseline NDDIE score and number of AED, and baseline NDDIE score and male gender. Specifically, increasing number of AEDs was associated with increased NDDIE, indicating a higher risk of depressive symptoms. Conversely, male gender was associated with decreased NDDIE score. No other factors were associated with NDDIE or MMSE at baseline or over time after correction for multiple comparisons.

Cognitive deficits are frequently seen in patients with epilepsy. The association is likely multifactorial. Age of onset of epilepsy as well as duration of epilepsy are likely relevant; patients with earlier age of onset and longer duration of epilepsy show worse memory performance. ${ }^{8,13}$ Anti-epileptic medications have also been shown to contribute to cognitive decline, ${ }^{10-12}$ poly-therapy being worse than mono-therapy, although number of seizure medications was not associated with MMSE in our data. However, we were limited by small sample size and our association, although not significant, was in the expected direction with increasing number of medications associated with a poor cognitive score. This direction could be due to patients on more anti-seizure medications being likely to have more severe epilepsy. However, we did not find a significant association between lower MMSE score and frequency of seizures in our study.

Studies have found correlations between BMI and cognitive status of patients ${ }^{20-22}$ in select populations. However, the evidence is not clear, and no definite association has been reported in patients with epilepsy. Although we found suggestion of an association between increasing BMI and decreasing MMSE score over 1 year, this association was not significant after correction for multiple comparisons and was a small clinical effect ( 0.06 decrease in MME for 1 unit increase in $\mathrm{BMI})$. Further investigation of this point with a larger sample size would be very interesting.

Epilepsy is also associated with psychosocial problems, including depressive symptoms. ${ }^{23}$ Part of this could be related to the severity of the epilepsy itself and also with how patients with epilepsy perceive themselves. ${ }^{24,25} \mathrm{~A}$ bidirectional relationship has been proposed between depression and epilepsy, which can be suggestive of some shared neurobiology between the two. ${ }^{3}$ Weatherburn et al. ${ }^{26}$ has proposed that the total burden of the co-morbid state is a contributor. They found that people with epilepsy have more co-morbidities than the general population, and depression increased with increasing co-morbidities. However, we did not find an association between either seizure frequency or disease duration and NDDIE in our study, although we were limited by our small sample size, patient report of seizure frequency, and disease duration. Depression is known to be more common in women across all age groups. ${ }^{27}$ Per our results, the gender gap seems to persist in people with epilepsy. Part of this could be because men tend to report sadness less than women on the scoring system, which has been pointed out in previous studies. ${ }^{26,28}$ It is interesting, though, that the gender gap seems to persist in people with epilepsy despite the additional co-morbidity of epilepsy, which adds work restrictions and life-style restrictions that may impact males more.

Anti-epileptic medications have been reported to cause cognitive and behavioral effects in epilepsy. ${ }^{11}$ As such, we were interested in whether we could identify particular medications that were more strongly associated with cognitive decline or mood. Among the medications we analyzed (levetiracetam, valproic acid, carbamazepine and lamotrigine), valproic acid use was possibly correlated with a higher depression score. This was surprising because given the side effect profile of these medications, one would expect patients on levetiracetam to have worse mood compared to those on other medications. Given that this association did not persist after correction for multiple comparisons, it may likely be due to chance. Additionally, we did not adjust for duration of medication use, which could have affected the results to some extent. However, this is data from real clinic patients and represents the situation that many clinicians face in deciding on treatment. Anti-epileptic medications have been reported to contribute to cognitive impairment in epilepsy. . $^{10-12}$ Among the medications analyzed, we did not find any significant correlation to cognitive score in our study. Again, however, we were limited by our small sample size and we may not have had adequate statistical power to detect these effects. Inclusion of anti-depressants did not markedly change the results of baseline or longitudinal analyses. The use of anti-depressant medications was found to be significant with respect to baseline NDDIE values. However, anti-depressants did not have significant effects for baseline MMSE values or any longitudinal analyses. It is worth noting that the proportion of patients taking anti-depressants is quite low at $4 \%$ of the patient population, which could limit the statistical power of detecting these effects.

Despite the innovative design of point-of-care standardized granular data on a clinic population, there are limitations to our study. We used NDDIE and MMSE scores for assessment, which are not a complete inventory for depression and cognitive status. The study also included only patients who could complete the score questionnaire for NDDIE and the MOCA or STMS tests. It did not include patients who 
could not complete these questionnaires or tests because they were non-English speaking and or too cognitively impaired to complete them. Also of note, we used NDDIE as a screening tool for depression and it is not a diagnostic tool and just indicative of depressive symptoms. However, it is unlikely that the bias introduced by this is differential. Thus, any impact on our findings is likely to result in a dilution of effect as opposed to introducing an association where none existed. Our population is also overwhelmingly Caucasian, so our findings are not generalizable to other racial/ethnic groups. Additionally, we did not have any specific medication exclusions, so the possibility of confounding by past medication use affected our results. Although we did include the use of anti-depressant medication in the analysis, the number of patients taking anti-depressants was low, limiting the statistical power of this analysis. While the physicians reviewed the use of all medications, the primary information regarding medication use was determined from patient report, which may be subject to some misclassification. Additionally, this was an observational trial in a clinic-based sample and not a randomized study. Therefore, we cannot exclude the possibility of unmeasured confounders skewing the observed associations. Lastly, we did have small sample sizes for some of the studied medications, thus we may have been underpowered to detect these effects. Despite these limitations, the methodology and novelty of this study provides the opportunity to further investigate these preliminary findings and provides an example of the opportunities for point-of-care research to potentially improve care for epilepsy patients. To improve upon these limitations, we will be continuing to collect data on more patients, including more races and ethnic groups, acquire valid cognitive assessment tools in other languages, and collect more definitive data on use of antidepressant medications.

\section{Acknowledgements}

The authors thank the medical assistants, nurses, neurologists, EMR Optimization and Enterprise Data Warehouse programmers, administrators, and research personnel at NorthShore University HealthSystem who contributed to the quality improvement and practice-based research initiative using the EMR. The authors thank Hannah Moulthrop, MS, for her assistance with editing, formatting, and submitting the manuscript for publication. Finally, the authors thank the epilepsy patients who inspire us to improve quality and to innovate our clinical practice every day.

Funding for this study was provided by the Auxiliary of NorthShore University HealthSystem with respect to the initial building of the tool kits and funding support from the Agency for Healthcare Research and Quality (RO1HS024057).

\section{Conflict of Interest}

The authors declare that they have no conflicts of interest.

\section{References}

1. Beghi E, Giussani G. Aging and the epidemiology of epilepsy. Neuroepidemiology 2018;51:216-23.

2. Salpekar JA, Mula M. Common psychiatric comorbidities in epilepsy: how big of a problem is it? Epilepsy Behav 2019;98(Pt B):293-7.

3. Hesdorffer DC, Ishihara L, Mynepalli L, Webb DJ, Weil J, Hauser WA. Epilepsy, suicidality, and psychiatric disorders: a bidirectional association. Ann Neurol 2012;72:184-91.

4. Whiteford HA, Ferrari AJ, Degenhardt L, Feigin V, Vos T. The global burden of mental, neurological and substance use disorders: an analysis from the Global Burden of Disease Study 2010. PLoS One 2015;10:e0116820.

5. Sander JW. The epidemiology of epilepsy revisited. Curr Opin Neurol 2003;16:165-70.

6. Ngugi AK, Bottomley C, Kleinschmidt I, Sander JW, Newton CR. Estimation of the burden of active and life-time epilepsy: a meta-analytic approach. Epilepsia 2010;51:883-90.

7. Mwangala PN, Kariuki SM, Nyongesa MK, et al. Cognition, mood and quality-of-life outcomes among low literacy adults living with epilepsy in rural Kenya: a preliminary study. Epilepsy Behav 2018;85:45-51.

8. Martin RC, Griffith HR, Faught E, Gilliam F, Mackey M, Vogtle L. Cognitive functioning in community dwelling older adults with chronic partial epilepsy. Epilepsia 2005;46:298-303.

9. Griffith HR, Martin RC, Bambara JK, Marson DC, Faught E. Older adults with epilepsy demonstrate cognitive impairments compared with patients with amnestic mild cognitive impairment. Epilepsy Behav 2006;8:161-8.

10. Eddy CM, Rickards HE, Cavanna AE. The cognitive impact of antiepileptic drugs. Ther Adv Neurol Disord 2011;4:385-407.

11. Loring DW, Meador KJ. Cognitive and behavioral effects of epilepsy treatment. Epilepsia 2001;42 Suppl 8:24-32.

12. Park SP, Kwon SH. Cognitive effects of antiepileptic drugs. J Clin Neurol 2008;4:99-106.

13. Miller LA, Galioto R, Tremont $G$, et al. Cognitive impairment in older adults with epilepsy: characterization and risk factor analysis. Epilepsy Behav 2016;56:113-7.

14. Maraganore DM, Frigerio R, Kazmi N, et al. Quality improvement and practice-based research in neurology using the electronic medical record. Neurol Clin Pract 2015;5:419-29.

15. Narayanan J, Dobrin S, Choi J, et al. Structured clinical documentation in the electronic medical record to improve quality and to support practice-based research in epilepsy. Epilepsia 2017;58:68-76.

16. Gilliam FG, Barry JJ, Hermann BP, Meador KJ, Vahle V, Kanner AM. Rapid 
detection of major depression in epilepsy: a multicentre study. Lancet Neurol 2006;5:399-405.

17. Kokmen E, Naessens JM, Offord KP. A short test of mental status: description and preliminary results. Mayo Clin Proc 1987;62:281-8.

18. Nasreddine ZS, Phillips NA, Bédirian V, et al. The Montreal Cognitive Assessment, MoCA: a brief screening tool for mild cognitive impairment. J Am Geriatr Soc 2005;53:695-9.

19. Tsoi KK, Chan JY, Hirai HW, Wong SY, Kwok TC. Cognitive tests to detect dementia: a systematic review and meta-analysis. JAMA Intern Med 2015; 175:1450-8.

20. Roalf DR, Moberg PJ, Xie SX, Wolk DA, Moelter ST, Arnold SE. Comparative accuracies of two common screening instruments for classification of Alzheimer's disease, mild cognitive impairment, and healthy aging. Alzheimers Dement 2013;9:529-37.

21. Calderón-Garcidueñas L, Jewells V, Galaz-Montoya C, et al. Interactive and additive influences of Gender, BMl and Apolipoprotein 4 on cognition in children chronically exposed to high concentrations of PM2.5 and ozone. APOE 4 females are at highest risk in Mexico City. Environ Res 2016;150:411-22.

22. Momtaz YA, Haron SA, Hamid TA, Ibrahim R, Tanjani PT. Body mass index (BMI) and cognitive functions in later life. Curr Alzheimer Res 2018;15:195-200.

23. Hassing $L B$, Dahl AK, Pedersen NL, Johansson B. Overweight in midlife is related to lower cognitive function 30 years later: a prospective study with longitudinal assessments. Dement Geriatr Cogn Disord 2010;29: 543-52.

24. Rai D, Kerr MP, McManus S, Jordanova V, Lewis G, Brugha TS. Epilepsy and psychiatric comorbidity: a nationally representative population-based study. Epilepsia 2012;53:1095-103.

25. Collings JA. Psychosocial well-being and epilepsy: an empirical study. Epilepsia 1990;31:418-26.

26. Weatherburn CJ, Heath CA, Mercer SW, Guthrie B. Physical and mental health comorbidities of epilepsy: population-based cross-sectional analysis of 1.5 million people in Scotland. Seizure 2017;45:125-31.

27. Kornstein SG. Gender differences in depression: implications for treatment. J Clin Psychiatry 1997;58 Supplement 15:12-8.

28. Barry LC, Allore HG, Guo Z, Bruce ML, Gill TM. Higher burden of depression among older women: the effect of onset, persistence, and mortality over time. Arch Gen Psychiatry 2008:65:172-8. 